\title{
Maximal regularity of delay equations in Banach spaces
}

\author{
by \\ Carlos Lizama and Verónica Poblete (Santiago)
}

\begin{abstract}
We characterize existence and uniqueness of solutions for an inhomogeneous abstract delay equation in Hölder spaces. The main tool is the theory of operatorvalued Fourier multipliers.
\end{abstract}

1. Introduction. Partial differential equations with delay have been extensively studied in the last years. In an abstract way they can be written as

$$
u^{\prime}(t)=A u(t)+F u_{t}+f(t), \quad t \in \mathbb{R},
$$

where $(A, D(A))$ is an (unbounded) linear operator on a Banach space $X$, $u_{t}(\cdot)=u(t+\cdot)$ on $[-r, 0], r>0$, and the delay operator $F$ is supposed to belong to $\mathcal{B}(C([-r, 0], X), X)$.

First studies on equation (1.1) go back to J. Hale [8] and G. Webb [12]. A general and systematic study of linear delay equations with emphasis on the qualitative behavior and asymptotic properties can be found in the recent monograph by Bátkai and Piazzera [5]. See also [13]. The problem to find conditions for all solutions of (1.1) to be in the same space as $f$ arises naturally from recent studies on maximal regularity and their application to nonlinear problems in the theory of evolution equations; see the recent monograph by Denk-Hieber-Prüss [7] and references therein.

Recently, a significant progress has been made in finding sufficient conditions for operator-valued functions to be $C^{\alpha}$-Fourier multipliers (see [3]). In particular, in [4] the theory of operator-valued Fourier multipliers is applied to obtain results on the hyperbolicity of delay equations and in [9] to obtain stability of linear control systems in Banach spaces. Also in [10] existence and uniqueness of periodic solutions for equation (1.1) via $L^{p}$-Fourier multiplier theorems has recently been obtained.

2000 Mathematics Subject Classification: 34G10, 34K30, 47D06. groups.

Key words and phrases: Fourier multipliers, delay differential equations, $C_{0}$-semi-

The first author is partially supported by FONDECYT Grant \#1050084. 
In this paper we obtain necessary and sufficient conditions of well-posedness of the delay equation (1.1) in the Hölder spaces $C^{\alpha}(\mathbb{R}, X)(0<\alpha<1)$, under the condition that $X$ is a $B$-convex space. We stress that here $A$ is not necessarily the generator of a $C_{0}$-semigroup.

The Fourier multiplier approach allows us to give a direct treatment of the equation, in contrast with the approach using the correspondence between (1.1) and the solutions of the abstract Cauchy problem

$$
\mathcal{U}^{\prime}(t)=\mathcal{A} \mathcal{U}(t)+\mathcal{F}(t), \quad t \geq 0,
$$

where $\mathcal{A}=\left(\begin{array}{cc}A & F \\ 0 & d / d \sigma\end{array}\right)$. In the latter case the question of well-posedness of the delay equation reduces to the question whether or not the operator $(\mathcal{A}, D(\mathcal{A}))$ generates a $C_{0}$-semigroup; see $[5,6,11]$ and references therein.

2. Preliminaries. Let $X, Y$ be Banach spaces and let $0<\alpha<1$. We consider the spaces

$$
\dot{C}^{\alpha}(\mathbb{R}, X)=\left\{f: \mathbb{R} \rightarrow X: f(0)=0,\|f\|_{\alpha}<\infty\right\}
$$

normed by

$$
\|f\|_{\alpha}=\sup _{t \neq s} \frac{\|f(t)-f(s)\|}{|t-s|^{\alpha}} .
$$

Let $\Omega \subset \mathbb{R}$ be an open set. By $C_{\mathrm{c}}^{\infty}(\Omega)$ we denote the space of all $C^{\infty}$ functions in $\Omega \subseteq \mathbb{R}$ having compact support in $\Omega$.

We denote by $\mathcal{F} f$ or $\widetilde{f}$ the Fourier transform, i.e.

$$
(\mathcal{F} f)(s):=\int_{\mathbb{R}} e^{-i s t} f(t) d t \quad\left(s \in \mathbb{R}, f \in L^{1}(\mathbb{R}, X)\right) .
$$

Definition 2.1. Let $M: \mathbb{R} \backslash\{0\} \rightarrow \mathcal{B}(X, Y)$ be continuous. We say that $M$ is a $\dot{C}^{\alpha}$-multiplier if there exists a mapping $L: \dot{C}^{\alpha}(\mathbb{R}, X) \rightarrow \dot{C}^{\alpha}(\mathbb{R}, Y)$ such that

$$
\int_{\mathbb{R}}(L f)(s)(\mathcal{F} \phi)(s) d s=\int_{\mathbb{R}}(\mathcal{F}(\phi \cdot M))(s) f(s) d s
$$

for all $f \in C^{\alpha}(\mathbb{R}, X)$ and $\phi \in C_{\mathrm{c}}^{\infty}(\mathbb{R} \backslash\{0\})$.

Here $(\mathcal{F}(\phi \cdot M))(s)=\int_{\mathbb{R}} e^{-i s t} \phi(t) M(t) d t \in \mathcal{B}(X, Y)$. Note that $L$ is well defined, linear and continuous (cf. [3, Definition 5.2]).

Define

$$
C^{\alpha}(\mathbb{R}, X)=\left\{f: \mathbb{R} \rightarrow X:\|f\|_{C^{\alpha}}<\infty\right\}
$$

with the norm

$$
\|f\|_{C^{\alpha}}=\|f\|_{\alpha}+\|f(0)\| .
$$

Let $C^{\alpha+1}(\mathbb{R}, X)$ be the Banach space of all $u \in C^{1}(\mathbb{R}, X)$ such that $u^{\prime} \in C^{\alpha}(\mathbb{R}, X)$, equipped with the norm

$$
\|u\|_{C^{\alpha+1}}=\left\|u^{\prime}\right\|_{C^{\alpha}}+\|u(0)\| \text {. }
$$


By Definition 2.1 and since

$$
\int_{\mathbb{R}}(\mathcal{F}(\phi M)(s))(s) d s=2 \pi(\phi M)(0)=0,
$$

it follows that $f \in C^{\alpha}(\mathbb{R}, X)$ implies $L f \in C^{\alpha}(\mathbb{R}, X)$. Moreover, if $f \in$ $C^{\alpha}(\mathbb{R}, X)$ is bounded then $L f$ is bounded as well (see [3, Remark 6.3]).

The following multiplier theorem is due to Arendt-Batty and $\mathrm{Bu}[3$, Theorem 5.3].

TheOREM 2.2. Let $M \in C^{2}(\mathbb{R} \backslash\{0\}, \mathcal{B}(X, Y))$ be such that

$$
\sup _{t \neq 0}\|M(t)\|+\sup _{t \neq 0}\left\|t M^{\prime}(t)\right\|+\sup _{t \neq 0}\left\|t^{2} M^{\prime \prime}(t)\right\|<\infty \text {. }
$$

Then $M$ is a $\dot{C}^{\alpha}$-multiplier.

Remark 2.3. If $X$ is $B$-convex, in particular if $X$ is a UMD space, Theorem 2.2 remains valid if condition (2.2) is replaced by the weaker condition

$$
\sup _{t \neq 0}\|M(t)\|+\sup _{t \neq 0}\left\|t M^{\prime}(t)\right\|<\infty
$$

where $M \in C^{1}(\mathbb{R} \backslash\{0\}, \mathcal{B}(X, Y))$ (cf. [3, Remark 5.5]).

We use the symbol $\widehat{f}(\lambda)$ for the Carleman transform:

$$
\widehat{f}(\lambda)= \begin{cases}\int_{0}^{\infty} e^{-\lambda t} f(t) d t, & \operatorname{Re} \lambda>0, \\ -\int_{-\infty}^{0} e^{-\lambda t} f(t) d t, & \operatorname{Re} \lambda<0\end{cases}
$$

where $f \in L_{\text {loc }}^{1}(\mathbb{R}, X)$ is of subexponential growth; by this we mean

$$
\int_{-\infty}^{\infty} e^{-\varepsilon|t|}\|f(t)\| d t<\infty \quad \text { for each } \varepsilon>0 .
$$

We remark that if $u^{\prime} \in L_{\text {loc }}^{1}(\mathbb{R}, X)$ is of subexponential growth, then

$$
\widehat{u^{\prime}}(\lambda)=\lambda \widehat{u}(\lambda)-u(0), \quad \operatorname{Re} \lambda \neq 0
$$

3. A characterization. In this section we consider the equation

$$
u^{\prime}(t)=A u(t)+F u_{t}+f(t), \quad t \in \mathbb{R},
$$

where $A: D(A) \subseteq X \rightarrow X$ is a closed linear operator, $f \in C^{\alpha}(\mathbb{R}, X)$, and, for some $r>0, F: C([-r, 0], X) \rightarrow X$ is a bounded linear operator. Moreover $u_{t}$ is an element of $C([-r, 0], X)$ defined by $u_{t}(\theta)=u(t+\theta)$ for $-r \leq \theta \leq 0$. 
EXAmple 3.1. Let $\mu:[-r, 0] \rightarrow \mathcal{B}(X)$ be of bounded variation. Let $F: C([-r, 0], X) \rightarrow X$ be the bounded operator given by the RiemannStieltjes integral

$$
F(\phi)=\int_{-r}^{0} \phi d \mu \quad \text { for all } \phi \in C([-r, 0], X) .
$$

An important special case involves operators $F$ defined by

$$
F(\phi)=\sum_{k=0}^{n} C_{k} \phi\left(\tau_{k}\right), \quad \phi \in C([-r, 0], X),
$$

where $C_{k} \in \mathcal{B}(X)$ and $\tau_{k} \in[-r, 0]$ for $k=0,1, \ldots, n$. For concrete equations with the above classes of delay operators see the monograph of Bátkai and Piazzera [5, Chapter 3].

Definition 3.2. We say that (1.1) is $C^{\alpha}$-well posed if for each $f \in$ $C^{\alpha}(\mathbb{R}, X)$ there is a unique function $u \in C^{\alpha+1}(\mathbb{R}, X) \cap C^{\alpha}(\mathbb{R},[D(A)])$ such that (1.1) is satisfied. by

Set $e_{\lambda}(t):=e^{i \lambda t}$ for all $\lambda \in \mathbb{R}$, and define the operators $\left\{F_{\lambda}\right\}_{\lambda \in \mathbb{R}} \subseteq \mathcal{B}(X)$

$$
F_{\lambda} x=F\left(e_{\lambda} x\right) \quad \text { for all } \lambda \in \mathbb{R} \text { and } x \in X .
$$

We define the real spectrum of (3.1) by

$$
\sigma(\Delta)=\left\{s \in \mathbb{R}: i s I-F_{s}-A \in \mathcal{B}([D(A)], X) \text { is not invertible }\right\} .
$$

Proposition 3.3. Let $X$ be a Banach space and let $A: D(A) \subset X \rightarrow X$ be a closed linear operator. Suppose that (1.1) is $C^{\alpha}$-well posed. Then

(i) $\sigma(\Delta)=\emptyset$,

(ii) $\left\{i \eta\left(i \eta I-A-F_{\eta}\right)^{-1}\right\}_{\eta \in \mathbb{R}}$ is bounded.

Proof. Let $x \in D(A)$ and let $u(t)=e^{i \eta t} x$ for $\eta \in \mathbb{R}$. Then $u_{t}(s)=$ $e^{i t \eta} e^{i s \eta} x$. Thus

$$
F\left(u_{t}\right)=e^{i t \eta} F\left(e_{\eta} x\right)=e^{i t \eta} F_{\eta} x .
$$

Now if $\left(i \eta-A-F_{\eta}\right) x=0$, then $u(t)$ is a solution of equation (1.1) when $f \equiv 0$. Hence by uniqueness $x=0$. Now let $L: C^{\alpha}(\mathbb{R}, X) \rightarrow C^{\alpha+1}(\mathbb{R}, X)$ be the bounded operator which takes each $f \in C^{\alpha}(\mathbb{R}, X)$ to the unique solution $u \in C^{\alpha+1}(\mathbb{R}, X)$ of (1.1). Fix $y \in X$ and $s_{0} \in \mathbb{R}$, and define $f(t)=e^{i t \eta} y$, $t \in \mathbb{R}$. Let $u(t)$ be the unique solution of (1.1) such that $L(f)=u$.

We claim that $v(t):=u\left(t+s_{0}\right)$ and $w(t):=e^{i \eta s_{0}} u(t)$ both satisfy (1.1) when $f$ is replaced by $e^{i s_{0} \eta} f(t)$. First we notice that

$$
v_{t}(s)=u\left(t+s_{0}+s\right)=u_{t+s_{0}}(s) .
$$


Hence $F\left(v_{t}\right)=F\left(u_{t+s_{0}}\right)$. Then an easy computation shows that $v(t)$ satisfies (1.1). On the other hand,

$$
w_{t}(s)=w(t+s)=e^{i \eta s_{0}} u(t+s)=e^{i \eta s_{0}} u_{t}(s) .
$$

Hence $F\left(w_{t}\right)=e^{i s_{0} \eta} F\left(u_{t}\right)$. Thus

$$
e^{i \eta s_{0}} u^{\prime}(t)=e^{i \eta s_{0}}\left(A u(t)+F\left(u_{t}\right)+f(t)\right)=A w(t)+F\left(w_{t}\right)+e^{i \eta s_{0}} f(t),
$$

that is, $w(t)$ satisfies (1.1). By uniqueness we again have

$$
u(t+s)=e^{i \eta s} u(t)
$$

for all $t, s \in \mathbb{R}$. In particular, when $t=0$ we obtain

$$
u(s)=e^{i \eta s} u(0), \quad s \in \mathbb{R} .
$$

Now let $x=u(0) \in D(A)$. Then $u(t)=e^{i \eta t} x$ satisfies (1.1), that is, by $(3.3)$,

$$
i \eta u(t)=A u(t)+F\left(u_{t}\right)+e^{i \eta t} y=A u(t)+e^{i \eta t} F_{\eta} x+e^{i \eta t} y .
$$

In particular, if $t=0$ we obtain

$$
i \eta x=A x+F_{\eta} x+y
$$

since $x=u(0)$. Thus

$$
\left(i \eta I-A-F_{\eta}\right) x=y
$$

and hence $i \eta I-A-F_{\eta}$ is bijective. This shows assertion (i) of the proposition.

Next we notice that $u(t)=\left(i \eta-A-F_{\eta}\right)^{-1} y$ by (3.4). Since $\left\|e_{\eta} \otimes x\right\|_{\alpha}=$ $K_{\alpha}|\eta|^{\alpha}\|x\|$, we have

$$
\begin{gathered}
K_{\alpha}|\eta|^{\alpha}\left\|i \eta\left(i \eta-A-F_{\eta}\right)^{-1} y\right\|=\left\|e_{\eta} \otimes i \eta\left(i \eta-A-F_{\eta}\right)^{-1} y\right\|_{\alpha}=\left\|u^{\prime}\right\|_{\alpha} \\
\leq\|u\|_{1+\alpha}=\|L f\|_{1+\alpha} \leq\|L\|\|f\|_{\alpha} \leq\|L\|\left(\|f\|_{\alpha}+\|f(0)\|\right) \\
=\|L\|\left(\left\|e_{\eta} \otimes y\right\|_{\alpha}+\|y\|\right) \leq\|L\|\left(K_{\alpha}|\eta|^{\alpha}+1\right)\|y\| .
\end{gathered}
$$

Hence for $\varepsilon>0$ it follows that

$$
\sup _{|\eta|>\varepsilon}\left\|i \eta\left(i \eta-A-F_{\eta}\right)^{-1} y\right\| \leq\|L\| \sup _{|\eta|>\varepsilon}\left(1+\frac{1}{K_{\alpha}|\eta|^{\alpha}}\right)<\infty .
$$

Recall that a Banach space $X$ has Fourier type $p$, where $1 \leq p \leq 2$, if the Fourier transform defines a bounded linear operator from $L^{p}(\mathbb{R}, X)$ to $L^{q}(\mathbb{R}, X)$, where $q$ is the conjugate index of $p$. For example, the space $L^{p}(\Omega)$, where $1 \leq p \leq 2$, has Fourier type $p$; $X$ has Fourier type 2 if and only if $X$ is a Hilbert space; $X$ has Fourier type $p$ if and only if $X^{*}$ has Fourier type $p$. Every Banach space has Fourier type $1 ; X$ is $B$-convex if it has Fourier type $p$ for some $p>1$. Every uniformly convex space is $B$-convex.

Our main result in this paper establishes that the converse of Proposition 3.3 is true. 
TheOREm 3.4. Let $A$ be a closed linear operator defined on a B-convex space $X$. Then the following assertions are equivalent:

(i) Equation (1.1) is $C^{\alpha}$-well posed.

(ii) $\sigma(\Delta)=\emptyset$ and $\sup _{\eta \in \mathbb{R}}\left\|i \eta\left(i \eta I-A-F_{\eta}\right)^{-1}\right\|<\infty$.

Proof. (ii) $\Rightarrow\left(\right.$ i). Define the operator $M(t)=\left(B_{t}-A\right)^{-1}$, with $B_{t}=$ it $I-F_{t}$. Note that by hypothesis $M \in C^{1}(\mathbb{R}, \mathcal{B}(X,[D(A)]))$.

We claim that $M$ is a $C^{\alpha}$-multiplier. In fact, by hypothesis it is clear that $\sup _{t \in \mathbb{R}}\|M(t)\|<\infty$. On the other hand, we have

$$
M^{\prime}(t)=-M(t) B_{t}^{\prime} M(t)
$$

with $B_{t}^{\prime}=i I-F_{t}^{\prime}$ and $F_{t}^{\prime}(x)=F\left(e_{t}^{\prime} x\right)$ where $e_{t}^{\prime}(s)=i s e^{i s t}$. Note that for each $x \in X$,

$$
\left\|F_{t} x\right\|_{X} \leq\left\|F\left(e_{t} x\right)\right\|_{X} \leq\|F\|\left\|e_{t} x\right\|_{\infty} \leq\|F\|\|x\|_{X},
$$

and

$$
\left\|F_{t}^{\prime} x\right\|_{X} \leq\left\|F\left(e_{t}^{\prime} x\right)\right\|_{X} \leq\|F\|\left\|e_{t}^{\prime} x\right\|_{\infty} \leq r\|F\|\|x\|_{X} .
$$

Hence $B_{t}^{\prime}$ is uniformly bounded with respect to $t \in \mathbb{R}$ and we conclude from the hypothesis that

$$
\sup _{t \in \mathbb{R}}\left\|t M^{\prime}(t)\right\|=\sup _{t \in \mathbb{R}}\left\|[t M(t)] B_{t}^{\prime} M(t)\right\|<\infty,
$$

and hence the claim follows from Theorem 2.2 and Remark 2.3.

Now, define $N \in C^{1}(\mathbb{R}, \mathcal{B}(X))$ by $N(t)=(i d \cdot M)(t)$, where $i d(t):=i t$ for all $t \in \mathbb{R}$. We will prove that $N$ is a $C^{\alpha}$-multiplier. In fact, with a direct calculation, we have

$$
\begin{aligned}
t N^{\prime}(t) & =i t M(t)+i t^{2} M^{\prime}(t)=i t M(t)+i[i t M(t)] B_{t}^{\prime}[i t M(t)] \\
& =N(t)+i N(t) B_{t}^{\prime} N(t) .
\end{aligned}
$$

By hypothesis and (3.6) it follows that

$$
\sup _{t \in \mathbb{R}}\left\|t N^{\prime}(t)\right\| \leq \sup _{t \in \mathbb{R}}\|N(t)\|+\sup _{t \in \mathbb{R}}\left\|N(t) B_{t}^{\prime} N(t)\right\|<\infty,
$$

hence from Theorem 2.2 and Remark 2.3 the claim is proved.

A similar calculation proves that $P \in C^{1}(\mathbb{R} \backslash\{0\}, \mathcal{B}(X))$ defined by $P(t)=F_{t} M(t)$ is a $C^{\alpha}$-multiplier. In fact, we have $t P^{\prime}(t)=F_{t}^{\prime} N(t)+$ $F_{t} t M^{\prime}(t)$, and hence from (3.5), (3.6) and (3.7) we see that $\sup _{t \in \mathbb{R}}\|P(t)\|+$ $\sup _{t \in \mathbb{R}}\left\|t P^{\prime}(t)\right\|<\infty$.

Let $f \in C^{\alpha}(\mathbb{R}, X)$. Since $M, N$ and $P$ are $C^{\alpha}$-multipliers, there exist $\bar{u} \in C^{\alpha}(\mathbb{R},[D(A)]), v \in C^{\alpha}(\mathbb{R}, X)$ and $w \in C^{\alpha}(\mathbb{R}, X)$ such that 


$$
\begin{aligned}
\int_{\mathbb{R}} \bar{u}(s)(\mathcal{F} \phi)(s) d s & =\int_{\mathbb{R}} \mathcal{F}(\phi \cdot M)(s) f(s) d s, \\
\int_{\mathbb{R}} v(s)(\mathcal{F} \psi)(s) d s & =\int_{\mathbb{R}} \mathcal{F}(\psi \cdot i d \cdot M)(s) f(s) d s, \\
\int_{\mathbb{R}} w(s)(\mathcal{F} \varphi)(s) d s & =\int_{\mathbb{R}} \mathcal{F}(\varphi \cdot F . M)(s) f(s) d s,
\end{aligned}
$$

for all $\phi, \psi, \varphi \in C_{\mathrm{c}}^{\infty}(\mathbb{R})$.

Note that for $x \in X$ and $\phi \in C_{\mathrm{c}}^{\infty}(\mathbb{R})$ we have

$$
\mathcal{F}(\phi F . M)(s) x=\int_{\mathbb{R}} e^{-i s t} \phi(t) F_{t} M(t) x d t=\int_{\mathbb{R}} e^{-i s t} \phi(t) F\left(e_{t} M(t) x\right) d t,
$$

where $\int_{\mathbb{R}} e^{-i s t} \phi(t) e_{t} M(t) x d t \in C([-r, 0], X)$. Now, for all $\theta \in[-r, 0]$ we have

$$
\left\|\int_{\mathbb{R}} e^{-i s t} \phi(t) e_{t}(\theta) M(t) x d t\right\|_{X} \leq \int_{\mathbb{R}}|\phi(t)|\|M(t) x\|_{X} d t .
$$

Since $F$ is bounded, we deduce that

$$
\mathcal{F}(\phi \cdot F . M)(s) x=F(\mathcal{F}(\phi \cdot e . M)(s) x) .
$$

Furthermore, observe that for $\theta \in[-r, 0]$ fixed we have $e .(\theta) \phi \in C_{\mathrm{c}}^{\infty}(\mathbb{R})$. Using (3.8) we obtain

$$
\begin{aligned}
\int_{\mathbb{R}} \bar{u}(s+\theta)(\mathcal{F} \phi)(s) d s & =\int_{\mathbb{R}} \bar{u}(s+\theta) \int_{\mathbb{R}} e^{-i s t} \phi(t) d t d s \\
& =\int_{\mathbb{R}} \bar{u}(s+\theta) \int_{\mathbb{R}} e^{-i(s+\theta) t} e_{t}(\theta) \phi(t) d t d s \\
& =\int_{\mathbb{R}} \bar{u}(s+\theta)(\mathcal{F} e .(\theta) \phi)(s+\theta) d s \\
& =\int_{\mathbb{R}} \bar{u}(s)(\mathcal{F} e .(\theta) \phi)(s) d s \\
& =\int_{\mathbb{R}} \mathcal{F}(e .(\theta) \phi \cdot M)(s) f(s) d s,
\end{aligned}
$$

hence $\int_{\mathbb{R}} \bar{u}_{s}(\mathcal{F} \phi)(s) d s=\int_{\mathbb{R}} \mathcal{F}(e . \phi \cdot M)(s) f(s) d s$.

Since $\theta \mapsto \int_{\mathbb{R}} \bar{u}_{s}(\theta)(\mathcal{F} \phi)(s) d s \in C([-r, 0], X)$ (see [3, p. 25]), from the boundedness of $F$ and (3.12) it follows that

$$
\begin{aligned}
\int_{\mathbb{R}} \mathcal{F}(\phi \cdot F . M)(s) f(s) d s & =\int_{\mathbb{R}} F \mathcal{F}(\phi \cdot e . M)(s) f(s) d s \\
& =\int_{\mathbb{R}} F \bar{u}_{s}(\mathcal{F} \phi)(s) d s
\end{aligned}
$$


for all $\phi \in C_{\mathrm{c}}^{\infty}(\mathbb{R})$. Since F.M is a $C^{\alpha}$-multiplier, from (3.10) we obtain

$$
\int_{\mathbb{R}} w(s)(\mathcal{F} \phi)(s) d s=\int_{\mathbb{R}} F \bar{u}_{s}(\mathcal{F} \phi)(s) d s
$$

for all $\phi \in C_{\mathrm{c}}^{\infty}(\mathbb{R})$. We conclude that there exists $y_{1} \in X$ satisfying $w(t)=$ $F \bar{u}_{t}+y_{1}$, proving that $F \bar{u} . \in C^{\alpha}(\mathbb{R}, X)$.

Choosing $\phi=i d \cdot \psi$ in (3.8) we deduce from (3.9) that

$$
\int_{\mathbb{R}} \bar{u}(s) \mathcal{F}(i d \cdot \psi)(s) d s=\int_{\mathbb{R}} v(s)(\mathcal{F} \psi)(s) d s,
$$

and it follows from Lemma 6.2 in [3] that $\bar{u} \in C^{\alpha+1}(\mathbb{R}, X)$ and $\bar{u}^{\prime}=v+y_{2}$ for some $y_{2} \in X$.

Since $(i d I-F .-A) M=I$ we have $i d \cdot M=I+F . M+A M$ and replacing in $(3.9)$ gives

$$
\begin{aligned}
\int_{\mathbb{R}} v(s)(\mathcal{F} \phi)(s) d s= & \int_{\mathbb{R}} \mathcal{F}(\phi \cdot(I+F . M+A M))(s) f(s) d s \\
= & \int_{\mathbb{R}}(\mathcal{F} \phi)(s) f(s) d s+\int_{\mathbb{R}} \mathcal{F}(\phi \cdot F . M)(s) f(s) d s \\
& +\int_{\mathbb{R}} \mathcal{F}(\phi \cdot A M)(s) f(s) d s
\end{aligned}
$$

for all $\phi \in C_{\mathrm{c}}^{\infty}(\mathbb{R})$.

Since $\bar{u}(t) \in D(A)$ and $\mathcal{F}(\phi \cdot M)(s) x \in D(A)$ for all $x \in X$, using the fact that $A$ is closed and inserting (3.8) and (3.13) in (3.15) we obtain

$$
\begin{aligned}
\int_{\mathbb{R}} v(s)(\mathcal{F} \phi)(s) d s= & \int_{\mathbb{R}} F \bar{u}_{s}(\mathcal{F} \phi)(s) d s+\int_{\mathbb{R}} A \bar{u}(s)(\mathcal{F} \phi)(s) f(s) d s \\
& +\int_{\mathbb{R}} f(s)(\mathcal{F} \phi)(s) d s
\end{aligned}
$$

for all $\phi \in C_{\mathrm{c}}^{\infty}(\mathbb{R})$. By Lemma 5.1 in [3] this implies that for some $y_{3} \in X$ one has

$$
v(t)=F \bar{u}_{t}+A \bar{u}(t)+f(t)+y_{3}, \quad t \in \mathbb{R} .
$$

Consequently, $\bar{u}^{\prime}(t)=v(t)+y_{2}=F \bar{u}_{t}+A \bar{u}(t)+f(t)+y$ where $y=$ $y_{2}+y_{3}$. In particular $A \bar{u} \in C^{\alpha}(\mathbb{R}, X)$. Now, by hypothesis we can define $x=(A+F)^{-1} y \in D(A)$, and then it is clear that $u(t):=\bar{u}(t)+x$ is in $C^{\alpha+1}(\mathbb{R}, X) \cap C^{\alpha}(\mathbb{R},[D(A)])$ and satisfies (1.1). We have shown that a solution of (1.1) exists.

In order to prove uniqueness, suppose that

$$
u^{\prime}(t)=A u(t)+F u_{t}, \quad t \in \mathbb{R},
$$

where $u \in C^{\alpha+1}(\mathbb{R}, X) \cap C^{\alpha}(\mathbb{R},[D(A)])$ and, as shown, $A u, F u . \in C^{\alpha}(\mathbb{R}, X)$. 
We claim that $\widehat{u} .(\lambda) \in C([-r, 0], X)$ for $\operatorname{Re} \lambda \neq 0$. In fact, let $\operatorname{Re} \lambda>0$. Then

$$
\begin{aligned}
\left\|e^{-\lambda t} u_{t}\right\|_{\infty} & =\sup _{\theta \in[-r, 0]}\left\|e^{-\lambda t} u(t+\theta)\right\|_{X} \leq \sup _{\theta \in[-r, 0]} e^{-\operatorname{Re} \lambda t}\left(1+|t+\theta|^{\alpha}\right) \\
& \leq e^{-\operatorname{Re} \lambda t}\left(1+(|t|+r)^{\alpha}\right) .
\end{aligned}
$$

Since $e^{-\operatorname{Re} \lambda t}\left(1+(|t|+r)^{\alpha}\right) \in L^{1}\left(\mathbb{R}_{+}\right)$, applying the dominated convergence theorem we obtain the claim. Analogously we argue for $\operatorname{Re} \lambda<0$.

Now, note that for $\operatorname{Re} \lambda>0$ and $\theta \in[-r, 0]$,

$$
\begin{aligned}
\int_{0}^{\infty} e^{-\lambda t} u_{t}(\theta) d t & =\int_{0}^{\infty} e^{-\lambda t} u(t+\theta) d t=\int_{\theta}^{\infty} e^{-\lambda(t-\theta)} u(t) d t \\
& =e^{\lambda \theta} \int_{\theta}^{\infty} e^{-\lambda t} u(t) d t=e^{\lambda \theta}\left(\int_{0}^{\infty} e^{-\lambda t} u(t) d t+\int_{\theta}^{0} e^{-\lambda t} u(t) d t\right) \\
& =e^{\lambda \theta} \widehat{u}(\lambda)+e^{\lambda \theta} \int_{\theta}^{0} e^{-\lambda t} u(t) d t .
\end{aligned}
$$

Analogously if $\operatorname{Re} \lambda<0$ and $\theta \in[-r, 0]$, then

$$
\begin{aligned}
-\int_{-\infty}^{0} e^{-\lambda t} u_{t}(\theta) d t & =-\int_{-\infty}^{0} e^{-\lambda t} u(t+\theta) d t=-\int_{-\infty}^{\theta} e^{-\lambda(t-\theta)} u(t) d t \\
& =-e^{\lambda \theta}\left(\int_{-\infty}^{0} e^{-\lambda t} u(t) d t-\int_{\theta}^{0} e^{-\lambda t} u(t) d t\right) \\
& =e^{\lambda \theta} \widehat{u}(\lambda)+e^{\lambda \theta} \int_{\theta}^{0} e^{-\lambda t} u(t) d t
\end{aligned}
$$

Since $F$ is bounded, we obtain

$$
\widehat{F u} .(\lambda)=F \widehat{u} .(\lambda)=F g \widehat{u}(\lambda)+F g h \quad \text { for } \operatorname{Re} \lambda \neq 0
$$

where $g(\theta)=e^{\lambda \theta}$ and $h(\theta)=\int_{\theta}^{0} e^{-\lambda t} u(t) d t$. Note that $g h \in C([-r, 0], X)$.

Since $\widehat{u^{\prime}}(\lambda)=\lambda \widehat{u}(\lambda)-u(0)$ for $\operatorname{Re} \lambda \neq 0$, one has $\widehat{u}(\lambda) \in D(A)$ and

$$
\widehat{u^{\prime}}(\lambda)=\widehat{A u}(\lambda)+\widehat{F u} .(\lambda) \text { for } \operatorname{Re} \lambda \neq 0 \text {. }
$$

Using the fact that $A$ is closed, from (3.18) and (3.19) we get

$$
(\lambda I-F g-A) \widehat{u}(\lambda)=u(0)+F g h \quad \text { for all } \lambda \in \mathbb{C} \backslash i \mathbb{R} .
$$

Since $i \mathbb{R} \subset \varrho(A)$, it follows that the Carleman spectrum $\operatorname{sp}_{C}(u)$ of $u$ is empty. Hence $u \equiv 0$ by [2, Theorem 4.8.2].

We denote by $\mathcal{K}_{F}(X)$ the class of operators in $X$ satisfying (ii) in the above theorem. If $A \in \mathcal{K}_{F}(X)$ we have $u^{\prime}, A u, F u . \in C^{\alpha}(\mathbb{R}, X)$, and hence we deduce the following result. 
Corollary 3.5. Let $X$ be $B$-convex and $A \in \mathcal{K}_{F}(X)$. Then

(i) (1.1) has a unique solution in $Z:=C^{\alpha+1}(\mathbb{R}, X) \cap C^{\alpha}(\mathbb{R},[D(A)])$ if and only if $f \in C^{\alpha}(\mathbb{R}, X)$.

(ii) There exists a constant $M>0$ independent of $f \in C^{\alpha}(\mathbb{R}, X)$ such that

$$
\left\|u^{\prime}\right\|_{C^{\alpha}(\mathbb{R}, X)}+\|A u\|_{C^{\alpha}(\mathbb{R}, X)}+\|F u .\|_{C^{\alpha}(\mathbb{R}, X)} \leq M\|f\|_{C^{\alpha}(\mathbb{R}, X) .}
$$

REMARK 3.6. The inequality (3.20) is a consequence of the closed graph theorem and known as the maximal regularity property for equation (1.1). From it we deduce that the operator $L$ defined by

$$
D(L)=Z, \quad(L u)(t)=u^{\prime}(t)-A u(t)-F u_{t},
$$

is an isomorphism onto. In fact, since $A$ is closed, the space $Z$ becomes a Banach space under the norm

$$
\|u\|_{Z}:=\|u\|_{C^{\alpha}(\mathbb{R}, X)}+\left\|u^{\prime}\right\|_{C^{\alpha}(\mathbb{R}, X)}+\|A u\|_{C^{\alpha}(\mathbb{R}, X)} .
$$

Such isomorphisms are crucial for the treatment of nonlinear versions of (1.1).

Assume $X$ is $B$-convex and $A \in \mathcal{K}_{F}(X)$ and consider the semilinear problem

$$
u^{\prime}(t)=A u(t)+F u_{t}+f(t, u(t)), \quad t \geq 0 .
$$

Define the Nemytskiu superposition operator $N: Z \rightarrow C^{\alpha}(\mathbb{R}, X)$ by $N(v)(t)=f(t, v(t))$, and the bounded linear operator

$$
S: C^{\alpha}(\mathbb{R}, X) \rightarrow Z
$$

by $S(g)=u$ where $u$ is the unique solution of the linear problem

$$
u^{\prime}(t)=A u(t)+F u_{t}+g(t) .
$$

Then to solve (3.21) we have to show that the operator $H: Z \rightarrow Z$ defined by $H=S N$ has a fixed point.

For related information we refer to Amann [1] where results on quasilinear delay equations involving the method of maximal regularity are presented.

We finish this paper with the following result which gives us a useful criterion to verify condition (ii) in the above theorem.

Theorem 3.7. Let $X$ be a $B$-convex space and let $A: D(A) \subset X \rightarrow X$ be a closed linear operator such that $i \mathbb{R} \subset \varrho(A)$ and $\sup _{s \in \mathbb{R}}\left\|A(i s I-A)^{-1}\right\|=$ : $M<\infty$. Suppose that

$$
\|F\|<\frac{1}{\left\|A^{-1}\right\| M}
$$

Then for each $f \in C^{\alpha}(\mathbb{R}, X)$ there is a unique function $u \in C^{\alpha+1}(\mathbb{R}, X) \cap$ $C^{\alpha}(\mathbb{R},[D(A)])$ such that $(1.1)$ is satisfied. 
Proof. From the identity

$$
i s I-A-F_{s}=(i s I-A)\left(I-F_{s}(i s I-A)^{-1}\right), \quad s \in \mathbb{R},
$$

it follows that $i s I-A-F_{s}$ is invertible whenever $\left\|F_{s}(i s I-A)^{-1}\right\|<1$. Next observe that

$$
\left\|F_{s}\right\| \leq\|F\|
$$

and hence

$$
\left\|F_{s}(i s I-A)^{-1}\right\|=\left\|F_{s} A^{-1} A(i s I-A)^{-1}\right\| \leq\|F\|\left\|A^{-1}\right\| M=: \alpha .
$$

Therefore, under the condition (3.22) we obtain $\sigma(\Delta)=\emptyset$ and the identity

$$
\begin{aligned}
\left(i s I-A-F_{s}\right)^{-1} & =(i s I-A)^{-1}\left(I-F_{s}(i s I-A)^{-1}\right) \\
& =(i s I-A)^{-1} \sum_{n=0}^{\infty}\left[F_{s}(i s I-A)^{-1}\right]^{n} .
\end{aligned}
$$

For all $n \in \mathbb{N}$ we have

$$
\begin{aligned}
& \left\|i s(i s I-A)^{-1}\left[F_{s}(i s I-A)^{-1}\right]^{n}\right\| \\
& \quad \leq\left\|i s(i s I-A)^{-1}\right\|\left\|F_{s} A^{-1} A(i s I-A)^{-1}\right\|^{n} \\
& \quad \leq\left\|i s(i s I-A)^{-1}\right\| F_{s} A^{-1}\left\|^{n}\right\| A(i s I-A)^{-1} \|^{n} \\
& \quad \leq\left\|i s(i s I-A)^{-1}\right\|\left\|A^{-1}\right\|^{n}\left\|F_{s}\right\|^{n}\left\|A(i s I-A)^{-1}\right\|^{n} .
\end{aligned}
$$

By (3.23) we obtain

$$
\begin{aligned}
\left\|i s(i s I-A)^{-1}\left[F_{s}(i s I-A)^{-1}\right]^{n}\right\| & \leq\left\|i s(i s I-A)^{-1}\right\|\left\|A^{-1}\right\|^{n}\|F\|^{n} M^{n} \\
& =\left\|i s(i s I-A)^{-1}\right\| \alpha^{n} .
\end{aligned}
$$

Finally, by (3.24), one has

$$
\left\|i s\left(i s I-A-F_{s}\right)^{-1}\right\| \leq\left\|i s(i s I-A)^{-1}\right\| \frac{1}{1-\alpha} \leq \frac{M+1}{1-\alpha} .
$$

This proves that $\left\{i s\left(i s I-A-F_{s}\right)^{-1}\right\}$ is bounded and the conclusion follows from Theorem 3.4.

\section{References}

[1] H. Amann, Quasilinear parabolic functional evolution equations, preprint.

[2] W. Arendt, C. Batty, M. Hieber and F. Neubrander, Vector-Valued Laplace Transforms and Cauchy Problems, Monogr. Math. 96, Birkhäuser, Basel, 2001.

[3] W. Arendt, C. Batty and S. Bu, Fourier multipliers for Hölder continuous functions and maximal regularity, Studia Math. 160 (2004), 23-51.

[4] A. Bátkai, E. Fašanga and R. Shvidkoy, Hyperbolicity of delay equations via Fourier multipliers, Acta Sci. Math. (Szeged) 69 (2003), 131-145.

[5] A. Bátkai and S. Piazzera, Semigroups for Delay Equations, Res. Notes Math. 10, A.K. Peters, Boston, MA, 2005. 
[6] A. Bátkai and S. Piazzera, Semigroups and linear partial differential equations with delay, J. Math. Anal. Appl. 264 (2001), 1-20.

[7] R. Denk, M. Hieber and J. Prüss, R-boundedness, Fourier multipliers and problems of elliptic and parabolic type, Mem. Amer. Math. Soc. 166 (2003), no. 788.

[8] J. K. Hale, Functional Differential Equations, Appl. Math. Sci. 3, Springer, 1971.

[9] Y. Latushkin and F. Räbiger, Operator valued Fourier multipliers and stability of strongly continuous semigroups, Integral Equations Operator Theory 51 (2005), 375-394.

[10] C. Lizama, Fourier multipliers and periodic solutions of delay equations in Banach spaces, J. Math. Anal. Appl., to appear.

[11] M. Stein, H. Vogt and J. Voigt, The modulus semigroup for linear delay equations III, J. Funct. Anal. 220 (2005), 388-400.

[12] G. Webb, Functional differential equations and nonlinear semigroups in $L^{p}$-spaces, J. Differential Equations 29 (1976), 71-89.

[13] J. Wu, Theory and Applications of Partial Differential Equations, Appl. Math. Sci. 119, Springer, 1996.

Departamento de Matemática

Facultad de Ciencias

Universidad de Santiago de Chile

Casilla 307, Correo 2

Santiago, Chile

E-mail: clizama@lauca.usach.cl

vpoblete@lauca.usach.cl

Received November 16, 2005

Revised version March 6, 2006 